\title{
Efficacy and safety of pemetrexed maintenance chemotherapy for advanced non-small cell lung cancer in a real-world setting
}

\author{
Jianjie Li", Yujia Chi", Guang Cao", Jun Zhao, Tongtong An, Meina Wu, Yuyan Wang, Minglei Zhuo, Xue \\ Yang, Bo Jia, Hanxiao Chen, Jingjing Wang, Xiaoyu Zhai, Ziping Wang \\ Key Laboratory of Carcinogenesis and Translational Research (Ministry of Education/Beijing), Department of Thoracic Medical Oncology, Peking \\ University Cancer Hospital \& Institute, Beijing, China \\ Contributions: (I) Conception and design: Z Wang, J Li; (II) Administrative support: Z Wang, J Li; (III) Provision of study materials or patients: J Li, \\ T An, M Wu, Y Wang, M Zhuo; (IV) Collection and assembly of data: J Li, Y Chi; (V) Data analysis and interpretation: J Li, G Cao; (VI) Manuscript \\ writing: All authors; (VII) Final approval of manuscript: All authors. \\ "These authors contributed equally to this work. \\ Correspondence to: Ziping Wang. Key Laboratory of Carcinogenesis and Translational Research (Ministry of Education/Beijing), Department \\ of Thoracic Medical Oncology, Peking University Cancer Hospital \& Institute, 52 Fucheng Road, Haidian District, Beijing 100142, China. \\ Email: wangzp2007@126.com.
}

Background: Pemetrexed maintenance therapy offers a survival benefit in patients with nonprogressive advanced nonsquamous non-small cell lung cancer (NSCLC) with good tolerability. This study was designed to analyze the efficacy and safety of pemetrexed maintenance chemotherapy in advanced nonsquamous NSCLC patients in a real-world setting.

Methods: The response rate (RR) and adverse events in 71 nonsquamous NSCLC patients treated with pemetrexed-based chemotherapy were observed until disease progression or unacceptable toxicities. Measures of survival were analyzed during follow-up.

Results: Of 69 efficacy-evaluable patients, the objective response rate (ORR) was $46.4 \%$ and the disease control rate (DCR) was $98.6 \%$. ORR showed no significant difference between patients who received pemetrexed as first-line therapy and those who received pemetrexed as second-line or higher treatment. The median treatment cycle for all patients was 8 . The median progression-free survival (PFS) was 9.5 months (m) and median overall survival (OS) was $30.5 \mathrm{~m}$. The univariate and multivariate analyses showed that the number of chemotherapy cycles was an independent factor for PFS. The most common adverse reactions were grade 1 to 2 hematologic toxicities, gastrointestinal reactions, and liver enzyme abnormalities. Only 1 patient experienced a grade 3 gastrointestinal event.

Conclusions: Pemetrexed maintenance chemotherapy can improve PFS in patients with advanced nonsquamous NSCLC with good tolerability.

Keywords: Pemetrexed; maintenance; non-small cell lung cancer (NSCLC); progression-free survival (PFS); adverse events

Submitted Dec 30, 2020. Accepted for publication Mar 17, 2021.

doi: $10.21037 /$ jtd-21-337

View this article at: http://dx.doi.org/10.21037/jtd-21-337 


\section{Introduction}

Lung cancer remains the leading cause of cancer incidence and mortality worldwide, with non-small cell lung cancer (NSCLC) accounting for $85 \%$ of cases. Most NSCLC patients are at an advanced stage of the disease at diagnosis (1). Although immune checkpoint inhibitors and targeted agents have shown encouraging treatment efficacy for NSCLC (2-4), platinum-based dual-drug chemotherapy is an integral part of first-line standard treatment for most patients with advanced nonsquamous NSCLC $(5,6)$. The phase III PARAMOUNT study demonstrated that in patients with advanced nonsquamous NSCLC who had nonprogressive disease (PD) after induction chemotherapy with 4 cycles of first-line pemetrexed plus cisplatin, pemetrexed continuation maintenance therapy significantly prolonged progression-free survival (PFS) (4.4 vs. 2.8 months) and overall survival (OS) (13.9 vs. 11.0 months) in patients with a performance status (PF) score of $0-1$ compared to placebo (7). Maintenance treatment is the prolongation of the treatment duration with the administration of additional drugs at the end of a defined number of initial chemotherapy cycles (8). Pemetrexed offers a high-efficacy and low-toxicity treatment option in the full management of advanced NSCLC, and the currently available clinical data support its use as first-line therapy, second-line therapy, maintenance therapy with the same drug, and maintenance therapy with different drugs $(7,9)$. Pemetrexed maintenance therapy has become a routine strategy in patients with advanced nonsquamous NSCLC. The therapy aims to prolong tumor response or stable disease (SD), and is started immediately after first-line (pemetrexed-containing platinum doublet) therapy. Recent research has supported that pemetrexed maintenance therapy improves progression-free survival (PFS) and overall survival (OS) with good tolerability and safety. Maintenance therapy is usually administered until disease progression or unacceptable toxicity (7).

Pemetrexed maintenance therapy has been recommended in the major guidelines, including the Chinese Society of Clinical Oncology (CSCO) guidelines, and it has been widely used in clinical settings. However, its role remains controversial due to limited survival benefit, additional drug toxicities, economic factors, and quality of life concerns. More data about the efficacy and safety of pemetrexed maintenance therapy in real world of China are necessary for the clinical outcomes analysis. Few studies have examined the outcomes of pemetrexed maintenance therapy in patients with driver gene mutations. Therefore, we retrospectively analyzed the clinical data of 71 patients with advanced NSCLC who had received platinum-based pemetrexed combinations and pemetrexed maintenance therapy in our center, with an attempt to determine the efficacy and safety of pemetrexed maintenance therapy in a real-world setting. We present the following article in accordance with the STROBE reporting checklist (available at http://dx.doi.org/10.21037/jtd-21-337).

\section{Methods}

\section{Subjects}

The clinical data of 71 patients with advanced NSCLC who had been treated in our center from January 2015 to June 2017 were retrospectively analyzed. There were 36 males and 35 females aged 37-76 years (median 60 years), among whom 36 patients $(50.7 \%)$ were $\geq 60$ years and $10(14.1 \%)$ were $\geq 70$ years. Pathologically, the vast majority of these patients had adenocarcinoma $(97.2 \%, 69 / 71)$, and there was only 1 case of large cell neuroendocrine carcinoma and 1 case of adenocarcinoma with neuroendocrine differentiation. The Eastern Collaborative Oncology Group (ECOG) performance status score was 0 in 27 cases (38.0\%), 1 in 35 cases (49.3\%), 2 in 3 cases, and 3 in 1 case. Thirtynine patients (54.9\%) had no history of smoking, 13 (18.3\%) had a history of previous smoking, and 17 (23.9\%) had not quit smoking. The tumors were in stage IIIA in 4 patients (5.6\%), stage IIIB in 3 patients $(4.2 \%)$, and in stage IV in the remaining 64 patients $(90.1 \%)$. Of the 71 patients, 22 (31.0\%) had epidermal growth factor receptor (EGFR) mutations, 5 (7.0\%) had anaplastic lymphoma kinase (ALK) fusions, and 3 had ROS proto-oncogene (ROS-1) fusions.

The inclusion criteria included the following: (I) with cytologically or histopathologically confirmed locally advanced or advanced inoperable stage III/IV NSCLC; (II) agreement to voluntarily undergo pemetrexed-based chemotherapy until disease progression or intolerable toxicity occurred, discontinuation according to the patients' own willingness, and signed informed consent prior to chemotherapy; (III) with adequate bone marrow function prior to chemotherapy (neutrophil count $>1.5 \times 10^{9} / \mathrm{L}$, platelet count $>100 \times 10^{9} / \mathrm{L}$, and hemoglobin $>10 \mathrm{~g} / \mathrm{L}$ ); (IV) adequate liver function (total bilirubin $<1.0$ time the upper limit of normal, glutamate transaminase $<1.5$ times the upper limit of normal, and glutamic oxalacetic transaminase $<1.5$ times the upper limit of normal); and (V) adequate 
renal function (serum creatinine level $<133 \mathrm{mmol} / \mathrm{L}$ and urea nitrogen $<8.3 \mathrm{mmol} / \mathrm{L}$ ).

The exclusion criteria included the following: (I) uncontrolled central nervous system metastasis; (II) presence of severe underlying cardiopulmonary disease; and (III) pregnancy or lactation.

\section{Treatment regimens}

All 71 patients had received pemetrexed-based chemotherapy regimens. Pemetrexed (Alimta, Lilly, USA) was administered at a dose of $500 \mathrm{mg} / \mathrm{m}^{2}$ on day 1 (every 21 days for 1 chemotherapy cycle). Pemetrexed was used as the first-line drug in 54 patients $(76.1 \%)$ and as a second-line or higherline drug in 17 patients (23.9\%). Fifteen of the patients treated with second-line pemetrexed had received first-line tyrosine kinase inhibitors (TKI). The treatment regimens included the following: (I) pemetrexed monotherapy in 5 cases (7.0\%); (II) dual-drug combinations with pemetrexed plus platinum in 50 cases, including the combinations with cisplatin in 31 cases $(43.7 \%)$, with carboplatin in 16 cases (22.5\%), and with nedaplatin in 3 cases (4.2\%); (III) tri-drug combinations (pemetrexed + platinum + anti-angiogenic drug) in 14 cases (19.7\%); (IV) pemetrexed combined with other treatments, including pemetrexed plus antiangiogenic drug in 1 case and pemetrexed plus antiangiogenic drug plus osimertinib in 1 case. Fifty-five patients (77.5\%) received pemetrexed maintenance therapy, the median number of cycles of maintenance therapy was 6 (range, 1-25 cycles), and the remaining 16 patients $(22.5 \%)$ did not receive pemetrexed maintenance therapy.

\section{Efficacy evaluation, adverse effects, and follow-up}

Medical histories and the results of physical examination, laboratory tests (including complete blood count, urinalysis, and liver and kidney function), and electrocardiography were collected before the administration of chemotherapy regimens. Baseline imaging data were available for all patients. The long diameters (for non-lymph node lesions) and short diameters (for lymph node lesions) of measurable target lesions were recorded, and the sites of nontarget lesions were recorded. Laboratory tests were performed every week, and all adverse events were collected. Patients were evaluated on imaging every 2 treatment cycles. Response evaluation was performed using the Response Evaluation Criteria in Solid Tumors (RECIST 1.1), which defines tumor response as complete response (CR), partial response (PR), stable disease (SD), or $\mathrm{PD}$. The objective response rate $(\mathrm{ORR})$ was the proportion of patients who achieved CR or PR, and the disease control rate (DCR) was defined as the proportion of patients with either an objective response (CR/PR) or SD. PFS was defined as the time elapsed from the start of the first chemotherapy cycle until disease progression or death of any cause, whichever occurred first. OS was the time from the start of the start of the first chemotherapy cycle until death of any cause (for patients lost to follow-up before death, the time of the last follow-up visit was calculated as the time of death). Adverse events were graded [1-5] according to the National Cancer Institute Common Terminology Criteria for Adverse Events, version 4.03. As 6 of 71 patients were lost to followup, the follow-up rate was $91.5 \%$. All subjects were followed up until June 2020, with a median follow-up period of 32 months (range, 5-72 months).

\section{Statistical analysis}

All data were analyzed by using the SPSS 20.0 software package (IBM Corp., USA). Count data are expressed as cases and percentages. The potential correlation between each clinical feature and PFS was analyzed by regression analysis, with PFS as the dependent variable and the clinical features as the independent variables. Survival measures were analyzed using the Kaplan-Meier method. Multivariate analysis of variance (ANOVA) was used to analyze the correlation between each clinical factor and PFS. A P value of $<0.05$ was considered significantly different.

\section{Ethics}

This study was approved by the Medical Ethics Committee of Peking University Cancer Hospital (No. 2018KT81). The study conformed to the provisions of the Declaration of Helsinki (as revised in 2013). All patients signed the "Informed Consent of Obtaining the Patient's Medical Records to Conduct A Scientific Study". The authors had no access to patient's identities.

\section{Results}

\section{Patient features and short-term efficacy}

A total of 71 patients with advanced nonsquamous NSCLC admitted to our center from January 2015 to June 2017 were included in this study, all of whom were treated with 


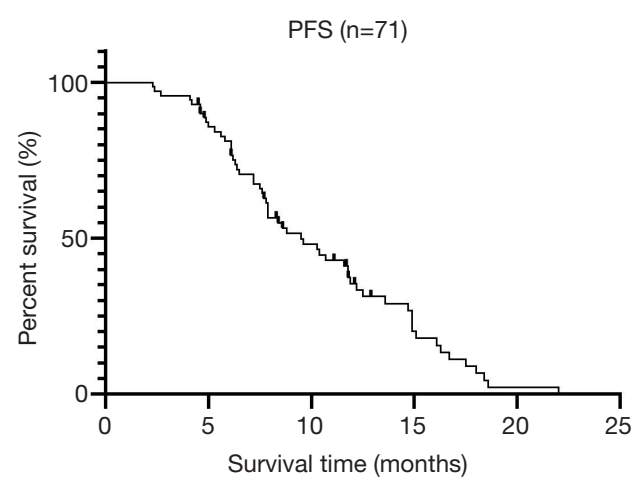

Figure 1 PFS survival curve of 71 patients with advanced NSCLC. PFS, progression-free survival; NSCLC, non-small cell lung cancer.

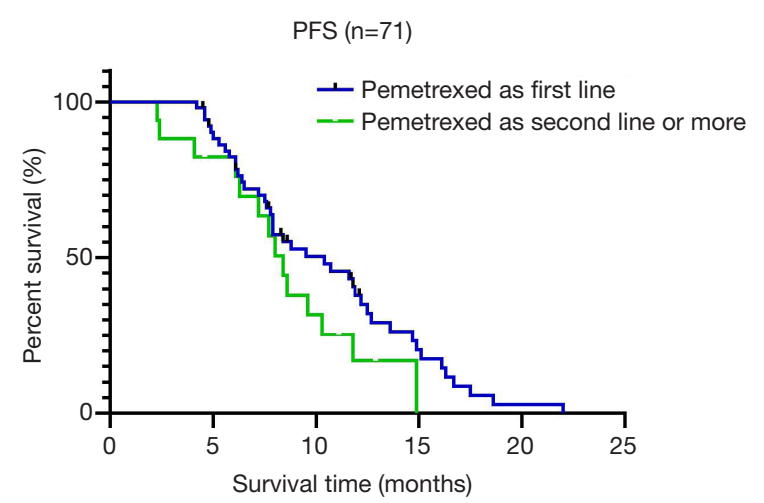

Figure 2 PFS survival curves of 71 patients with advanced NSCLC treated with pemetrexed as first-line versus $\geq$ second-line treatment $(\mathrm{n}=71)$. PFS, progression-free survival; NSCLC, non-small cell lung cancer.

pemetrexed-based regimens and could be evaluated for efficacy, safety, and survival.

Sixty-nine patients were efficacy-evaluable, among whom the treatment responses were as follows: CR, 0; PR, 32 (46.4\%); SD, 36 (52.2\%); and PD, 1 (1.4\%). The ORR was $46.4 \%(32 / 69)$ and the DCR was $98.6 \%(68 / 69)$. Of the 52 patients treated with first-line pemetrexed treatment, 25 achieved PR, with an ORR of $48.1 \%$ (25/52); of the 17 patients treated with second- and higher-line pemetrexed treatment, 7 achieved PR, with an ORR of $41.2 \%$ (7/17).

\section{PFS and OS after different lines of pemetrexed treatment}

\section{PFS}

The median number of treatment cycles was 8 (range, 3-31) in 71 patients, and the median PFS was 9.5 months (95\% CI: 6.3-10.7 months) (Figure 1). The median PFS was 10.4 months in 54 patients treated with first-line pemetrexed-based regimen and 8.4 months in 17 patients treated with the second- and higher-line pemetrexed-based regimen (among whom 15 patients received first-line EGFR TKI-targeted therapy) $(\mathrm{P}=0.145)$ (Figure 2). Thirty-nine patients (54.9\%) died within follow-up.

\section{Univariate and multivariate analyses of the predictors of PFS}

Univariate analysis showed that PFS did not significantly differ among patients with different age, gender, smoking history, ECOG score, driver gene mutations (EGFR, ALK, ROS-1, etc.), treatment lines, maintenance therapy, and anti-angiogenic drugs (all $\mathrm{P}>0.05$ ). Only two factors significantly affected PFS: number of the cycles of combination chemotherapy and pemetrexed maintenance therapy (Table 1). The multivariate analysis further confirmed that the number of chemotherapy cycles was an independent factor for PFS.

\section{OS}

The median OS was 30.5 months the in 71 patients. The median OS was 37.5 months in 54 patients treated with first-line pemetrexed-based regimen and 53.4 months in 17 patients treated with the second- and higher-line pemetrexed-based regimen (among whom 15 patients received first-line EGFR TKI-targeted therapy) $(\mathrm{P}=0.066)$ (Figure 3).

\section{Univariate and multivariate analyses of the predictors of OS}

Univariate analysis showed that OS did not significantly differ among patients with different sex, smoking history, driver gene mutations, treatment lines, pemetrexed maintenance therapy, and antiangiogenic therapy (all $\mathrm{P}>0.05$ ); however, patients with different age, ECOG scores, and number of pemetrexed treatment cycles showed significantly different median OS $(\mathrm{P}<0.05)$ (Table 2). The multivariate analysis failed to identify any independent factors for OS $(\mathrm{P}>0.05)$.

\section{Toxicities}

All 71 patients received 3 or more cycles of chemotherapy (range, 3-31). The common adverse reactions of the pemetrexed-based regimens were hematologic toxicities, 
Table 1 Univariate analysis of the predictors of PFS in 71 patients with advanced NSCLC

\begin{tabular}{|c|c|c|c|c|}
\hline Clinical characteristics & $\mathrm{N}$ & Median PFS (m) & $\chi^{2}$ value & $P$ value \\
\hline Age & & & 0.742 & 0.912 \\
\hline$\leq 60 \mathrm{y}$ & 33 & 10.3 & & \\
\hline $60 y$ & 38 & 7.9 & & \\
\hline Sex & & & 0.917 & 0.338 \\
\hline Male & 35 & 8.4 & & \\
\hline Female & 36 & 10.3 & & \\
\hline Smoking status & & & 0.154 & 0.901 \\
\hline No & 48 & 9.6 & & \\
\hline Yes & 23 & 9.5 & & \\
\hline ECOG PS & & & 1.007 & 0.315 \\
\hline 0 & 26 & 11.6 & & \\
\hline 1 & 40 & 7.9 & & \\
\hline$\geq 2$ & 5 & 7.7 & & \\
\hline Driver gene mutation & & & 1.073 & 0.300 \\
\hline Negative & 26 & 7.9 & & \\
\hline Positive & 40 & 9.6 & & \\
\hline Unknown & 5 & 13.6 & & \\
\hline Line of chemotherapy & & & 0.151 & 0.698 \\
\hline First & 54 & 9.5 & & \\
\hline Second or higher & 17 & 9.6 & & \\
\hline Cycles of chemotherapy & & & 9.641 & 0.002 \\
\hline$\leq 6$ & 27 & 6.1 & & \\
\hline $6-12$ & 31 & 10.3 & & \\
\hline$\geq 12$ & 13 & 13.6 & & \\
\hline Maintenance with chemc & thera & & 4.616 & 0.003 \\
\hline No & 16 & 6.1 & & \\
\hline Yes & 55 & 10.3 & & \\
\hline $\begin{array}{l}\text { Response to } \\
\text { chemotherapy }\end{array}$ & & & 0.058 & 0.809 \\
\hline PR & 32 & 9.5 & & \\
\hline $\mathrm{SD}$ & 36 & 9.6 & & \\
\hline \multicolumn{5}{|l|}{ Anti-VEGF therapy } \\
\hline No & 56 & 8.5 & 0.774 & 0.442 \\
\hline Yes & 15 & 7.5 & & \\
\hline
\end{tabular}

PFS, progression-free survival; NSCLC, non-small cell lung cancer; ECOG PS, Eastern Collaborative Oncology Group performance status; PR, partial response; SD, stable disease; VEGF, vascular endothelial growth factor.

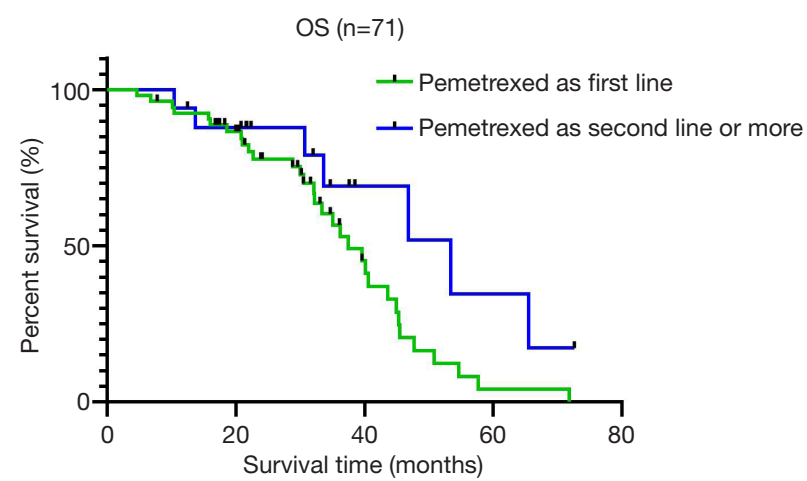

Figure 3 OS survival curves of 71 patients with advanced NSCLC treated with pemetrexed as first-line versus $\geq$ second-line treatment $(\mathrm{n}=71)$. OS, overall survival, NSCLC, non-small cell lung cancer.

mainly a grade $1-2$ decline in the total leukocyte counts (TLC) (27 patients, $38.0 \%$ ) and neutrophil counts (3 patients, $32.4 \%$ ). In addition, the incidences of anemia and thrombocytopenia were below $10 \%$. Among the nonhematologic toxicities, gastrointestinal reactions (28 patients, 39.4\%) and liver enzyme abnormalities (9 patients, $12.7 \%$ ) were predominant. All events were grade 1 or 2, except for a grade 3 event in 1 patient. Some rarer $(<5 \%)$ adverse reactions were grade 1 to 2 rash and peripheral neuritis (Tables 2 and 3).

\section{Discussion}

All 71 patients included in our current study received pemetrexed-based chemotherapy. Pathologically, the vast majority of these patients had adenocarcinoma (69/71, $97.2 \%$ ), with only 1 case of large cell neuroendocrine carcinoma and 1 case of adenocarcinoma with neuroendocrine differentiation. For patients with adenocarcinoma, pemetrexed-based regimens are the preferred first-line treatment in most guidelines. According to the JMDB study (10), a large phase III clinical trial comparing pemetrexed plus cisplatin versus gemcitabine plus cisplatin in patients with stage IIIB or IV NSCLC, there was no statistical difference in OS between these two groups; however, for patients with adenocarcinoma or large cell carcinoma, pemetrexed plus cisplatin was superior to gemcitabine plus cisplatin, with a respective median OS of 12.6 vs. 10.9 months [hazard ratio $(\mathrm{HR})=0.84 ; \mathrm{P}=0.033$ ] The pemetrexed plus cisplatin group also showed better tolerability and significantly lower incidences of grade 3-4 hematologic toxicities and febrile neutropenia. 
Table 2 Univariate analysis of the predictors of OS in 71 patients with advanced NSCLC

\begin{tabular}{|c|c|c|c|c|}
\hline Clinical characteristics & $\mathrm{n}$ & Median OS (m) & $\chi^{2}$ value & $P$ value \\
\hline Age & & & 5.542 & 0.019 \\
\hline$\leq 60 \mathrm{y}$ & 33 & 31.8 & & \\
\hline $60 y$ & 38 & 30.0 & & \\
\hline Sex & & & 1.175 & 0.278 \\
\hline Male & 35 & 29.8 & & \\
\hline Female & 36 & 30.7 & & \\
\hline Smoking status & & & 0.031 & 0.859 \\
\hline No & 48 & 40.6 & & \\
\hline Yes & 23 & 37.5 & & \\
\hline ECOG PS & & & 0.315 & 0.0007 \\
\hline 0 & 26 & 32.0 & & \\
\hline 1 & 40 & 30.2 & & \\
\hline$\geq 2$ & 5 & 15.5 & & \\
\hline Driver gene mutation & & & 1.007 & 0.946 \\
\hline Negative & 26 & 30.5 & & \\
\hline Positive & 40 & 29.6 & & \\
\hline Unknown & 5 & 35.1 & & \\
\hline Line of chemotherapy & & & 3.380 & 0.066 \\
\hline First & 54 & 37.5 & & \\
\hline Second or higher & 17 & 53.4 & & \\
\hline Cycles of chemotherapy & & & 6.211 & 0.013 \\
\hline$\leq 6$ & 27 & 30.5 & & \\
\hline $6-12$ & 31 & 44.9 & & \\
\hline$\geq 12$ & 13 & 43.6 & & \\
\hline \multicolumn{3}{|c|}{ Maintenance with chemotherapy } & 1.024 & 0.312 \\
\hline No & 16 & 30.0 & & \\
\hline Yes & 55 & 30.6 & & \\
\hline \multicolumn{3}{|c|}{ Response to chemotherapy } & 1.164 & 0.281 \\
\hline PR & 32 & 44.9 & & \\
\hline SD & 36 & 35.1 & & \\
\hline \multicolumn{5}{|l|}{ Anti-VEGF therapy } \\
\hline No & 56 & 30.5 & 0.262 & 0.609 \\
\hline Yes & 15 & 18.6 & & \\
\hline
\end{tabular}

OS, overall survival; ECOG PS, Eastern Collaborative Oncology Group performance status; PR, partial response; SD, stable disease; VEGF, vascular endothelial growth factor.
Table 3 Adverse events in 71 advanced NSCLC patients treated with pemetrexed

\begin{tabular}{|c|c|c|}
\hline Adverse event & CTCAE grade (V4.03) & $\mathrm{n}(\%)$ \\
\hline \multicolumn{3}{|l|}{ Hematologic } \\
\hline \multirow[t]{2}{*}{ Leukemia } & $1-2$ & $25(35.2)$ \\
\hline & $3-4$ & $2(2.8)$ \\
\hline \multirow[t]{2}{*}{ Neutropenia } & $1-2$ & 17 (23.9) \\
\hline & $3-4$ & $6(8.5)$ \\
\hline \multirow[t]{2}{*}{ Anemia } & $1-2$ & $5(7.0)$ \\
\hline & $3-4$ & $0(0)$ \\
\hline \multirow[t]{2}{*}{ Thrombocytopenia } & $1-2$ & $3(4.2)$ \\
\hline & $3-4$ & $0(0)$ \\
\hline \multicolumn{3}{|l|}{ Nonhematologic } \\
\hline \multirow[t]{2}{*}{ Digestive tract reactions } & $1-2$ & $27(38.0)$ \\
\hline & $3-4$ & $1(1.4)$ \\
\hline \multirow[t]{2}{*}{ ALT/AST increase } & $1-2$ & $9(12.7)$ \\
\hline & $3-4$ & $0(0)$ \\
\hline \multirow[t]{2}{*}{ Peripheral neurotoxicity } & $1-2$ & $3(4.2)$ \\
\hline & $3-4$ & $0(0)$ \\
\hline \multirow[t]{2}{*}{ Rash } & $1-2$ & $3(4.2)$ \\
\hline & $3-4$ & $0(0)$ \\
\hline
\end{tabular}

NSCLC, non-small cell lung cancer; CTCAE, Common Terminology Criteria for Adverse Events; ALT, alanine aminotransferase; AST, aspartate aminotransferase.

Among all efficacy-evaluable patients in our current study, the ORR after treatment with pemetrexed-based regimens was $46.4 \%$ (32/69) and the DCR was $98.5 \%$ (68/69), with no statistical difference in ORR between the first- and second-line treatments. The ORR and DCR were close to the findings in the retrospective and prospective studies. For instance, the ORR and DCR were $44 \%$ and $97 \%$, respectively in the pemetrexed group of the PARAMOUNT study (7). The efficacy analysis in our current study showed that 71 patients received a median of 8 cycles (range, 3-31 cycles) of pemetrexed, which yielded a median PFS of 9.5 months and a median survival of 30.5 months, which were superior to the ORR (30\%), median PFS (5 months), and median survival (12 months) reported in a previous study where first-line chemotherapy with platinum plus novel third-generation drugs were applied (11). Our findings were also consistent with the results of 
other studies on pemetrexed maintenance therapy. In the PARAMOUNT study (7), after 4 cycles of pemetrexed plus cisplatin induction therapy, progression-free patients continued pemetrexed maintenance therapy until the disease progressed or the therapy became intolerable; the mean number of maintenance cycles was 7.9 (range, 1-44) for pemetrexed, which significantly prolonged patients' PFS (median 4.4 vs. 2.8 months; $\mathrm{HR}=0.60 ; \mathrm{P}<0.001$ ) and OS (median 13.9 vs. 11.0 months; $\mathrm{HR}=0.78 ; \mathrm{P}=0.019,5$ ) compared with placebo. In the JMEN study (12), patients who had not progressed on 4 cycles of platinumbased chemotherapy were randomly assigned to receive pemetrexed plus best supportive care or placebo plus best supportive care until disease progression; the results showed that pemetrexed maintenance therapy significantly prolonged PFS (median 4.3 vs. 2.6 months; HR =0.50; $\mathrm{P}<0.0001$ ) and $\mathrm{OS}$ (median 13.4 vs. 10.6 months; $\mathrm{HR}=0.79$; $\mathrm{P}=0.012$ ) compared with placebo.

In our current study, ORR and PFS did not significantly differ between the anti-angiogenesis subgroup and the nonanti-angiogenesis subgroup. In the ECOG-ACRIN 5508 study (13), maintenance therapy with the combination of bevacizumab and pemetrexed achieved a median PFS of 7.5 months and a median OS of 16.4 months; in contrast, the median PFS and the median OS were 5.1 and 15.9 months in the pemetrexed monotherapy group and 3.2 and 14.4 months in the bevacizumab monotherapy groups, respectively. Bevacizumab plus pemetrexed significantly prolonged PFS compared with bevacizumab or pemetrexed maintenance therapy, with a nonsignificant OS benefit and a higher incidence of grade 3-4 toxicity. Thus, pemetrexed monotherapy maintenance or bevacizumab monotherapy maintenance remains the preferred option for monotherapy maintenance in patients with advanced nonsquamous NSCLC $(14,15)$.

Despite its retrospective design, our current study showed no statistical differences in ORR and PFS between patients receiving first- and second-line therapy, and most patients $(15 / 17)$ treated with second-line pemetrexed received targeted therapy in the first-line setting. Due to the presence of driver gene mutations, first-line oral targeted drugs did not affect the efficacy of secondline chemotherapy. There was prolonged OS in secondline settings due to oral administration of targeted drugs, although no statistical significant was noted due to the small sample size. Our study also found that 16 patients (22.5\%) did not receive pemetrexed maintenance therapy due to limited chemotherapy benefit, toxicity, and other reasons; rather, they adopted a watch-and-wait strategy during follow-up. A multicenter randomized phase III clinical trial in Norway (16) compared two groups of patients with advanced nonsquamous NSCLC after induction chemotherapy: one group was directly treated with pemetrexed maintenance therapy, and the other group was first observed and then administered with pemetrexed at progression. Patients in the maintenance group had longer OS (median 12.0 vs. 10.0 months; $\mathrm{P}=0.10$ ) and PFS (median 3.1 vs. 1.9 months; $\mathrm{P}<0.01$ ), which was consistent with our findings that the number of chemotherapy cycles was an independent prognostic factor for PFS. Although the results of prospective and retrospective studies have confirmed the survival benefit of maintenance therapy, some patients still choose to adopt a watch-and-wait strategy during followup and will be rechallenged after disease progression due to the limited benefits and adverse effects of the maintenance therapy. A retrospective study (17) analyzed 31 patients with nonsquamous NSCLC who achieved disease control with first-line pemetrexed-based regimens and who received pemetrexed-based chemotherapy again after disease progression and found that patients with $\mathrm{PFS} \geq 10$ months benefited from rechallenge. In the subgroup with PFS1 $<10$ months, 4 were EGFR-mutation positive and 13 received EGFR-TKI therapy, which yielded a median PFS2 was 5.38 months; in the subgroup with PFS $1 \geq 10$ months, 4 were EGFR-mutation positive and 9 received EGFRTKI therapy; the median PFS2 was 4.33 months, which was not significantly different from that in the subgroup with a PFS1 $<10$ months, suggesting that chemotherapy efficacy was not affected by EGFR-TKI treatment, as found in our current study.

The most common adverse reactions in our patients receiving pemetrexed-based chemotherapy were grade 1-2 hematologic toxicities, gastrointestinal reactions, and liver enzyme abnormalities, which were resolved after treatment, and no dose reduction of the chemotherapy was required. Only 1 patient experienced a grade 3 gastrointestinal event, which was considered to be related to cisplatin. Some rarer $(<5 \%)$ adverse reactions were grade $1-2$ rash and peripheral neuritis (Table 2). In general, the tolerability was good in all patients, and no dose reduction was required due to treatment-related toxicities.

In summary, our current study further demonstrated in a "real-world" setting that pemetrexed maintenance therapy could achieve longer PFS with good tolerability in patients with nonsquamous NSCLC regardless of driver gene status. Recently, immunotherapy and targeted 
therapy have achieved significant effects on non-squamous NSCLC patients. Pemetrexed maintenance therapy in non-squamous NSCLC provided significantly longer survival and infrequent severe adverse events. Pemetrexed combined with immunotherapy or targeted therapy maintenance therapy should be considered as further trial. Although pemetrexed maintenance therapy is an integral part of the multidisciplinary treatment for patients with advanced nonsquamous NSCLC, regimen selection should also consider factors including adherence, quality of life, and pharmacoeconomics in addition to survival benefit. Furthermore, the maintenance therapy and re-challenging programs should be tailored according to the patients' specific conditions.

\section{Acknowledgments}

Funding: Capital Clinical Characteristics and Application Research 211-02-099.

\section{Footnote}

Reporting Checklist: The authors have completed the STROBE reporting checklist. Available at http://dx.doi. org/10.21037/jtd-21-337

Data Sharing Statement: Available at http://dx.doi. org/10.21037/jtd-21-337

Conflicts of Interest: All authors have completed the ICMJE uniform disclosure form (available at http://dx.doi. org/10.21037/jtd-21-337). The authors have no conflicts of interest to declare.

Ethical Statement: The authors are accountable for all aspects of the work in ensuring that questions related to the accuracy or integrity of any part of the work are appropriately investigated and resolved. This study was approved by the Medical Ethics Committee of Peking University Cancer Hospital (No. 2018KT81). The study conformed to the provisions of the Declaration of Helsinki (as revised in 2013). All patients signed the "Informed Consent of Obtaining the Patient's Medical Records to Conduct A Scientific Study". The authors had no access to patient's identities.

Open Access Statement: This is an Open Access article distributed in accordance with the Creative Commons
Attribution-NonCommercial-NoDerivs 4.0 International License (CC BY-NC-ND 4.0), which permits the noncommercial replication and distribution of the article with the strict proviso that no changes or edits are made and the original work is properly cited (including links to both the formal publication through the relevant DOI and the license). See: https://creativecommons.org/licenses/by-nc-nd/4.0/.

\section{References}

1. Siegel RL, Miller KD, Jemal A. Cancer statistics, 2015. CA Cancer J Clin 2015;65:5-29.

2. Reck M, Rodríguez-Abreu D, Robinson AG, et al. Pembrolizumab versus Chemotherapy for PD-L1Positive Non-Small-Cell Lung Cancer. N Engl J Med 2016;375:1823-33.

3. Soria JC, Ohe Y, Vansteenkiste J, et al. Osimertinib in Untreated EGFR-Mutated Advanced Non-Small-Cell Lung Cancer. N Engl J Med 2018;378:113-25.

4. Peters S, Camidge DR, Shaw AT, et al. Alectinib versus Crizotinib in Untreated ALK-Positive Non-Small-Cell Lung Cancer. N Engl J Med 2017;377:829-38.

5. NSCLC Meta-Analyses Collaborative Group. Chemotherapy in addition to supportive care improves survival in advanced non-small-cell lung cancer: a systematic review and meta-analysis of individual patient data from 16 randomized controlled trials. J Clin Oncol 2008;26:4617-25.

6. Socinski MA, Schell MJ, Peterman A, et al. Phase III trial comparing a defined duration of therapy versus continuous therapy followed by second-line therapy in advancedstage IIIB/IV non-small-cell lung cancer. J Clin Oncol 2002;20:1335-43.

7. Paz-Ares LG, de Marinis F, Dediu M, et al. PARAMOUNT: Final overall survival results of the phase III study of maintenance pemetrexed versus placebo immediately after induction treatment with pemetrexed plus cisplatin for advanced nonsquamous non-small-cell lung cancer. J Clin Oncol 2013;31:2895-902.

8. Novello S, Milella M, Tiseo M, B, et al. Maintenance therapy in NSCLC: why? To whom? Which agent? J Exp Clin Cancer Res 2011;30:50.

9. Hanna N, Shepherd FA, Fossella FV, et al. Randomized phase III trial of pemetrexed versus docetaxel in patients with non-small-cell lung cancer previously treated with chemotherapy. J Clin Oncol 2004;22:1589-97.

10. Scagliotti GV, Parikh P, von Pawel J, et al. Phase III study comparing cisplatin plus gemcitabine with cisplatin 
plus pemetrexed in chemotherapy-naive patients with advanced-stage non-small-cell lung cancer. J Clin Oncol 2008;26:3543-51.

11. Schiller JH, Harrington D, Belani CP, et al. Comparison of four chemotherapy regimens for advanced non-smallcell lung cancer. N Engl J Med 2002;346:92-8.

12. Ciuleanu T, Brodowicz T, Zielinski C, et al. Maintenance pemetrexed plus best supportive care versus placebo plus best supportive care for non-small-cell lung cancer: a randomised, double-blind, phase 3 study. Lancet 2009;374:1432-40.

13. Ramalingam SS, Dahlberg SE, Belani CP, et al. Pemetrexed, Bevacizumab, or the Combination As Maintenance Therapy for Advanced Nonsquamous NonSmall-Cell Lung Cancer: ECOG-ACRIN 5508. J Clin Oncol 2019;37:2360-7.

14. Patel JD, Hensing TA, Rademaker A, et al. Phase II study of pemetrexed and carboplatin plus bevacizumab with maintenance pemetrexed and be vacizumab as first-line therapy for nonsquamous non-small-cell lung cancer. J

Cite this article as: Li J, Chi Y, Cao G, Zhao J, An T, Wu M, Wang Y, Zhuo M, Yang X, Jia B, Chen H, Wang J, Zhai X, Wang Z. Efficacy and safety of pemetrexed maintenance chemotherapy for advanced non-small cell lung cancer in a realworld setting. J Thorac Dis 2021;13(3):1813-1821. doi: 10.21037/ jtd-21-337
Clin Oncol 2009;27:3284-9.

15. Patel JD, Socinski MA, Garon EB, et al. PointBreak: a randomized phase III study of pemetrexed plus carboplatin and bevacizumab followed by maintenance pemetrexed and bevacizumab versus paclitaxel plus carboplatin and bevacizumab followed by maintenance bevacizumab in patients with stage IIIB or IV nonsquamous non-small-cell lung cancer. J Clin Oncol 2013;31:4349-57.

16. Halvorsen TO, Stokke K, Killingberg KT, et al. Randomized phase III trial comparing switch-maintenance pemetrexed with observation followed by pemetrexed at progression in advanced NSCLC. Acta Oncol 2020;59:1051-7.

17. Zhuo ML, Bai H, Wang ZJ, et al. Rechallenge with pemetrexed-based chemotherapy improves the survival of patients with advanced non-squamous non-small-cell lung cancer. Mol Clin Oncol 2014;2:953-9.

(English Language Editor: J. Gray) 